\title{
Inferring fault strength from earthquake rupture properties and the tectonic implications of high pore pressure faulting
}

\author{
Stephen A. Miller \\ Geophysics Institute, ETH-Zurich 8093 Switzerland \\ (Received January 4, 2002; Revised September 8, 2002; Accepted September 9, 2002)
}

\begin{abstract}
The strength of seismogenic faults is fundamental to earthquake mechanics and plate tectonics, affecting many subsidiary processes in the solid earth. The key to understanding fault strength is determining the pore pressures and hydraulic properties within the faults and surrounding crust. The debate has lasted over decades, with evidence provided for both strong fault and weak fault scenarios. A recently proposed hypothesis for quantifying the strength at which faults fail uses earthquake scaling arguments to show that earthquakes fail over a range of strengths in the upper 15-20 km, and at near-lithostatic pore pressure below this depth. This observation, if correct, has important implications for crustal hydraulics, plate tectonics, and earthquake hazard assessment. This paper summarizes the arguments for high pore pressure faulting, and explores its implications for earthquake stress drops, strength of the lithosphere, and earthquake scaling. The hope is to establish a general framework for quantifying the role of fluids in the earthquake process, and to demonstrate that high fluid pressure may dominate failure of the brittle crust.
\end{abstract}

\section{Introduction}

Plate kinematics, earthquake rupture properties, the strength of the lithosphere, and frictional heating all fundamentally depend on the strength of faults. Fault strength is defined here in terms of the shear stress necessary to initiate frictional sliding $\left(\tau_{f}\right)$, and is related to the effective confining stress by $\tau_{f}=\mu_{s} \sigma_{e}=\mu_{s}\left(\sigma_{n}-P_{p}\right)$; where the effective confining stress $\sigma_{e}$ is the normal stress $\sigma_{n}$ minus the pore pressure $P_{p}$ acting on the fault plane. If the fluid pressure approaches values comparable to the normal stress, then faults can fail under very low shear stress and behave as weak faults. If temperature-dependent deformation processes are not activated, then this relationship is independent of depth and faults can fail in a brittle manner to large depths (e.g. intermediate subduction zone earthquakes). Therefore, fault zone pore pressure has potential to be a dominant controlling factor in deformation and fracture of the brittle lithosphere. In stable and relatively homogeneous crust, few mechanisms exist to increase pore pressures much beyond hydrostatic because any increase in pore pressure will diffuse, leading to hydrostatic pore pressures. Indeed, studies of crustal pore pressures do show hydrostatic pore pressure conditions (Townend and Zoback, 2000; Manning and Ingebritsen, 1999), with (by implication) permeability between $10^{-16} \sim 10^{-17} \mathrm{~m}^{2}$. The behavior of active fault zones should be quite different from a stable setting because large-displacement faults usually contain gouge from tectonic wear. Gouge substantially reduces permeability, thus limiting diffusive processes in the fault zone and communication with the surrounding country rock. In the case of

Copy right(c) The Society of Geomagnetism and Earth, Planetary and Space Sciences (SGEPSS); The Seismological Society of Japan; The Volcanological Society of Japan; The Geodetic Society of Japan; The Japanese Society for Planetary Sciences. sedimentary basins, overpressures are generated by mechanical compaction (pressure solution), and can be maintained if overlain by an impermeable cap.

Dilatant slip associated with large earthquakes shatters the impermeable barrier, allowing fluid flow and fast diffusion to reduce fluid pressures. The pre-seismic low permeability fault zone switches to a post-seismic highly permeable channel for expulsion of mantle fluids and rapid in-plane pressure equilibration. This scenario is supported by many geophysical, geological, and geochemical observations. This scenario also directly affects the earthquake process because increasing fluid pressures in fault zones are at least as effective in bringing a fault to failure as stress increases from plate motion and stress transfer from surrounding slip events. If an impermeable cap or sealed fault zone hydraulically isolates overpressures developed from some generic fluid source (e.g. dehydration, compaction, etc.), then this weak zone may persist.

Much has been inferred about the behavior of fault zone fluids from inferences from fault zone drilling (Floyd et al., 2001), geochemical (Quattrocchi, 1999), and geologic observations (Evans and Chester, 1995). The prevailing view from these and other studies (Caine et al., 1996) is that faults behave as seals during quiescence that transform to co- and post-seismic channels through dilatant slip (Sibson, 1992; Cox, 1995; Miller et al., 1996). That is, fault pore pressure varies during the earthquake cycle and provides an important aspect to the entire earthquake process (Blanpied et al., 1992; Sleep and Blanpied, 1992). The earthquake can change the hydraulic properties in and near the fault zone and trigger fluid flow, while healing and sealing reduce the permeability over time and lead to increased fluid pressure given some source. Evidence of over-pressured fault zones is existent but scarce (Floyd et al., 2001). This is not to say 
that it is not common, only that very few large-scale studies have been completed with this focus.

The purpose of this paper is to propose a method for inferring fault pore pressure at depth by investigating the geometric and seismic moment properties of large earthquakes.

\section{Inferring Pore Pressures from Rupture Proper- ties}

The scaling relationship between the seismic moment $\left(M_{o}\right)$ and the rupture length $(L)$ of large earthquakes has been debated for years (Scholz, 1982, 1994; Romanowicz, 1992; Mai and Beroza, 2000; Shaw and Scholz, 2001; Romanowicz and Ruff, 2002). The issue comes down to two different views; either $M_{o} \propto L$ (the W-model) or $M_{o} \propto L^{2}$ (the L-model). In the $\mathrm{W}$-model, the average slip on the fault is controlled by the fault width, while in the L-model, the average slip is controlled by the fault length. An alternative hypothesis (Miller, 2002b) proposes that the relationship between the seismic moment and the fault length is also controlled by the strength of the fault, where strength is defined as the ratio of pore pressure to the normal stress on the fault.

Assuming that the normal stress on the fault is the lithostatic stress $\left(\sigma_{m}\right)$, the failure condition along a fault is;

$$
\tau_{f}=\mu_{s} \sigma_{e}=\mu_{s}\left(\sigma_{m}-P p\right)=\mu_{s} \rho_{r} g Z(1-\lambda)
$$

where $\sigma_{e}$ is the effective confining stress, and $\mu_{s}, \rho_{r}, g$, and $Z$, are, respectively, the static friction coefficient, rock density, gravity acceleration, and the average depth of the event. The dimensionless pore pressure $\lambda\left(\lambda=P_{f} / \sigma_{m}\right)$ ranges from $0.37<\lambda<1$, where the lower limit is hydrostatic, and the upper limit is lithostatic pore pressure.

Assuming a simple slip-weakening model for friction, when an earthquake initiates and the fault begins to slip, the stress drops from the initial static failure stress to the dynamic sliding stress. Therefore, the stress drop $\Delta \tau$ is;

$$
\Delta \tau=\left(\mu_{s}-\mu_{d}\right) \sigma_{e}=\left(\mu_{s}-\mu_{d}\right) \rho_{r} g Z(1-\lambda) .
$$

Linear elasticity shows that stress drop can be uniquely determined by the fault geometry, the slip and the shear modulus by (Scholz, 1990);

$$
\Delta \tau=C G\left(\frac{\bar{u}}{\Lambda}\right)
$$

where $\bar{u}$ is the mean slip, $G$ is the shear modulus, and $C$ is a constant that depends on the geometry of the rupture. The term in parentheses is the co-seismic strain change across the rupture, with $\Lambda$ the characteristic length. For a constant stress drop, the characteristic length $(\Lambda)$ is the crack radius for a circular rupture, the fault width $W$ in the $\mathrm{W}$ model and the fault length $L$ in the L-model. For small ruptures, the average slip grows linearly with the crack radius to maintain constant stress drop, resulting in $M_{o} \propto L^{3}$ (area scaling). For large strike-slip earthquakes, if average slip is controlled by the width of the rupture, then the slip stays constant independent of its length (W-model). However, it has been observed that slip increases with rupture length (Scholz, 1982), leading to the suggestion that $\Lambda$ is the larger dimension, or rupture length (L-model). A statistical analysis of strike-slip earthquakes (Romanowicz and Ruff, 2002) shows evidence for a cross over from area scaling to $\mathrm{W}$-model scaling at rupture lengths of about $75 \mathrm{~km}$, but as argued by Scholz (1994), there is no mechanistic explanation for such a cross-over distance. However, modeling results of more complicated rupture geometries and slip distributions do support such a cross-over distance (Yin and Rogers, 1996). Using a different approach of loading a fault plane in an elastic layer overlying a viscous asthenosphere, Mats'ura and Sato (1997) show a change in scaling due to the separate loading effects of viscous drag at the base of the lithosphere and linear loading due to dislocation pile-ups along the fault edge. The change in scaling reflects how slip is recovered in an earthquake from previous tectonic loading. Over an earthquake recurrence interval $T$, the slip along the fault should recover the plate displacement $\left(V_{p l} T\right)$ assuming stress drops inferred from large earthquakes $(\approx 2 \mathrm{MPa})$ by the relation:

$$
\bar{u}=\frac{\Delta \tau L}{G(\beta+\alpha L)}
$$

where $G$ is the shear modulus, $\bar{u}$ is the mean slip, $\alpha$ is the time-dependent effect of loading at the base, while $\beta$ is loading from the pile-up of edge dislocations on the fault plane. This model predicts a scaling change when $L$ grows large because slip at small $L$ is proportional to $L$, but is independent of $L$ at large rupture length. Both $\alpha$ and $\beta$ are determined from a model, with reported values of $\alpha \approx$ $1.4 \times 10^{-2} \mathrm{~km}^{-1}$ and $\beta \approx 1$ (Matsu'ura and Sato, 1997; Fujii and Matsu'ura, 2000).

The principal problem in all of the models is to determine the relationship between the stress drop and average slip of an earthquake. Recent modelling results of rupture properties along a strike-slip fault in a 3-dimensional elastic half-space (Miller, 2002a) show a relationship between stress drop and slip of the form,

$$
\bar{u}=\frac{\Delta \tau L}{G\left(1+\lambda \frac{L}{W}\right)} .
$$

The form of Eq. (5) is identical to Eq. (4) if the substitution $\alpha=\frac{\lambda}{W}$ is made. In both equations, the average slip is proportional to the stress drop times a geometric constant, with the geometric constant in Eq. (5) related to the strength of the fault supporting the rupture. In Eq. (4), the average slip is controlled by the combined contributions from the base loading and dislocation pile-ups along the fault plane. For small ruptures (e.g. $\frac{L}{W} \lesssim 3$ ), slip is controlled by the edge loading and increases linearly with $L$. As $L$ grows very large, slip is dominated by the base loading, and slip is independent of $L$. On the other hand, Eq. (5) describes the behavior of a propagating elastic dislocation spontaneously generated along a vertical fault plane under a very heterogeneous state of stress (Miller, 2002a). In analogy to the Matsu'ura and Sato model, the stress state through which the ruptures propagate is a result of continuous loading at the base of the elastic body combined with dislocation pile-ups of previous slip events along the fault plane.

Equation (5) says that slip is proportional to the rupture length out to some distance, asymptotically approaching constant slip for large $L$. Furthermore, the transition to 

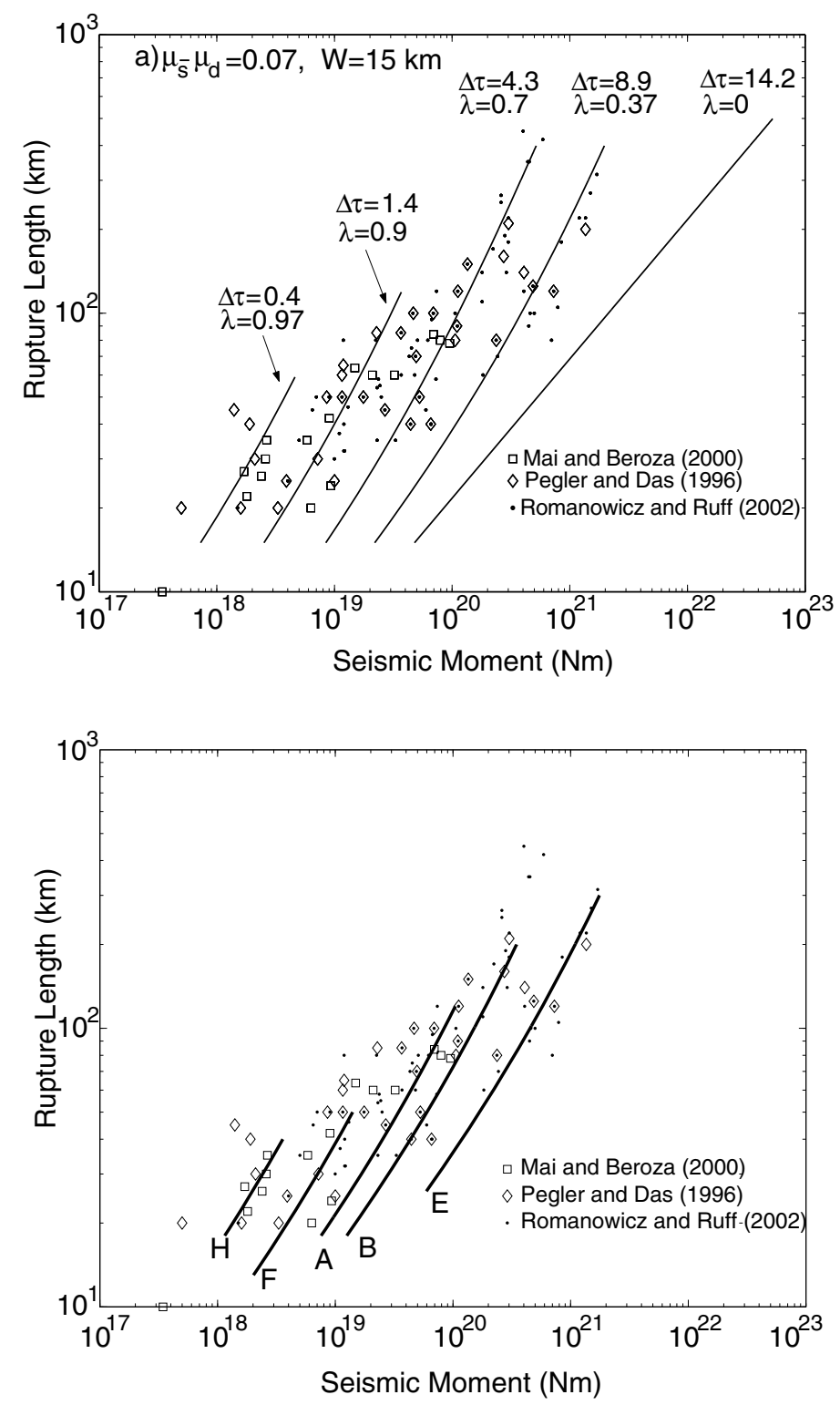

Fig. 1. (a) Eq. (6) superposed on the strike-slip earthquake catalogs for the case of $W=15 \mathrm{~km}$ and $\mu_{s}-\mu_{d}=0.07$. Stress drop in units of MPa. This plot shows that the data cannot be fit by a single line because the scatter is a reflection of the fault overpressure through which the rupture propagated. (b) Earthquake catalogs superposed with the synthetic moment-length catalogs from a fluid-controlled fault dislocation model. The letters in (b) indicate different model realizations, see (Miller, 2002a) for more detail.

constant slip is controlled by the fault strength $\lambda$. A physical explanation for this behavior can be argued as follows. Consider a rupture that has saturated at the seismogenic width and is constrained to propagate along strike only. As the rupture arrives at an un-slipped section, the slip associated with the initial stress drop at the rupture front determines the amplitude and length-scale of the stress transfer back to the already-slipping regions. For a small stress drop rupture front (e.g. small initial slip), the stress transfer feedback distance is limited, so the parts of the fault (at large distances behind the rupture front) that are slipping decelerate and eventually stop. Conversely, large stress drop at the rupture front (e.g. high initial slip) provides a longer range stress transfer to keep slipping regions slipping as the rupture propagates along the fault plane. Therefore, slip is an increasing function of rupture length but the function is dependent on the initial stress drop at the rupture front. Consequently, a high stress drop rupture front results in a higher average slip than a low stress drop. Ultimately, the rupture front is far enough downstream to no longer influence this slipping region; slip ceases and the average slip is constant with ongoing rupture. The hypothesis is that since stress drop at the rupture front controls the feedback distance and is itself a function of $\lambda$ (Eq. (1)), then the transition from $\bar{u}=f(L)$ to $\bar{u}=$ constant should also be a function of the same $\lambda$. It could also be argued that if a fault heals when the slip velocity reaches a healing threshold, then a high slip velocity requires more time to reach the healing threshold while an initial low slip velocity heals quite readily. In this case, since the slip velocity is a function of stress drop at the rupture front, it should also be controlled by the fault strength $(\lambda)$. In other models of rupture behavior, the spatial dimension of the slip band should be dependent on a rapid healing property at the rupture front (Cochard and Madariaga, 1994). 


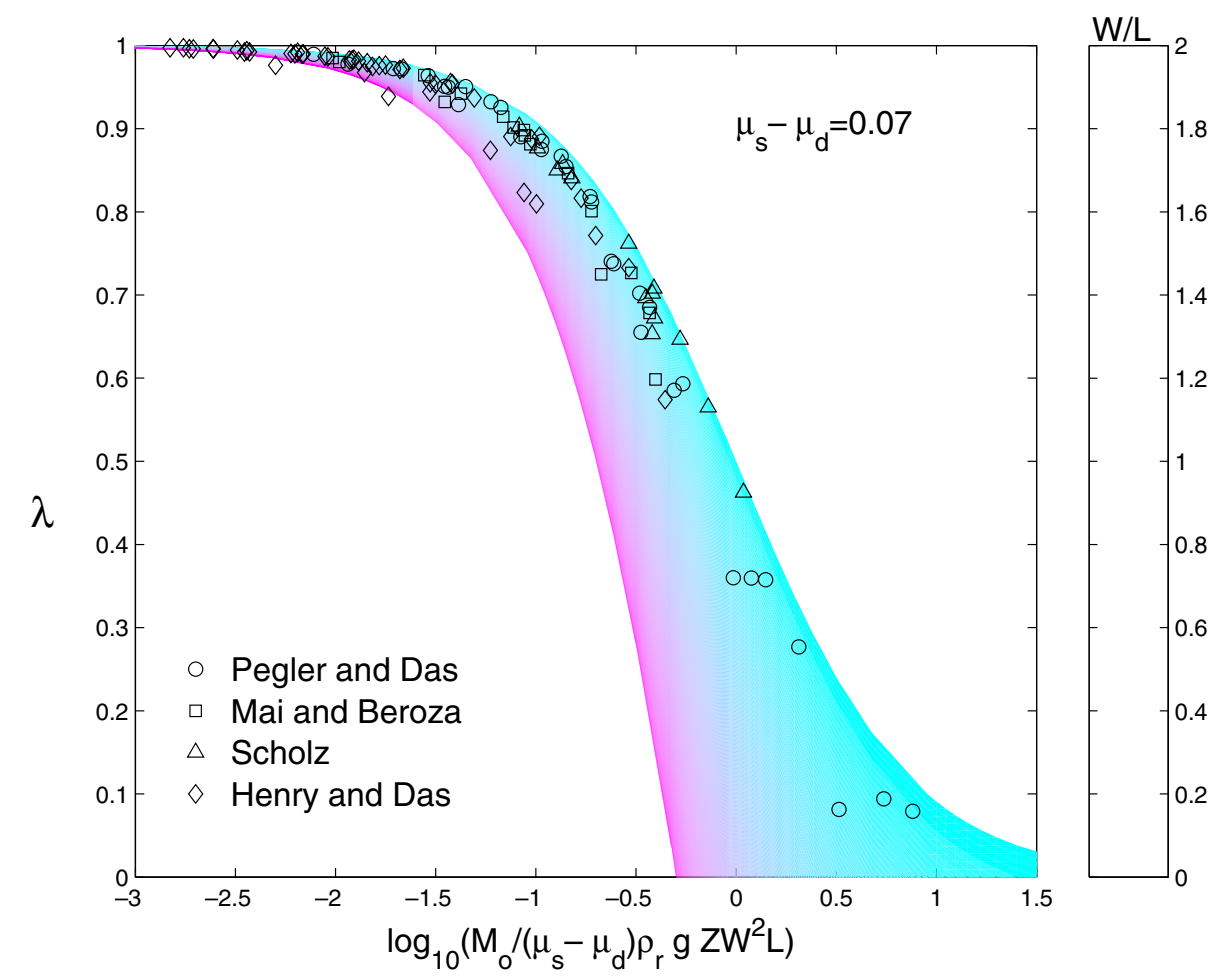

Fig. 2. Earthquake catalogs superposed on Eq. (7) showing that the data in Fig. 1 collapses to a single function. From Miller (2002b).

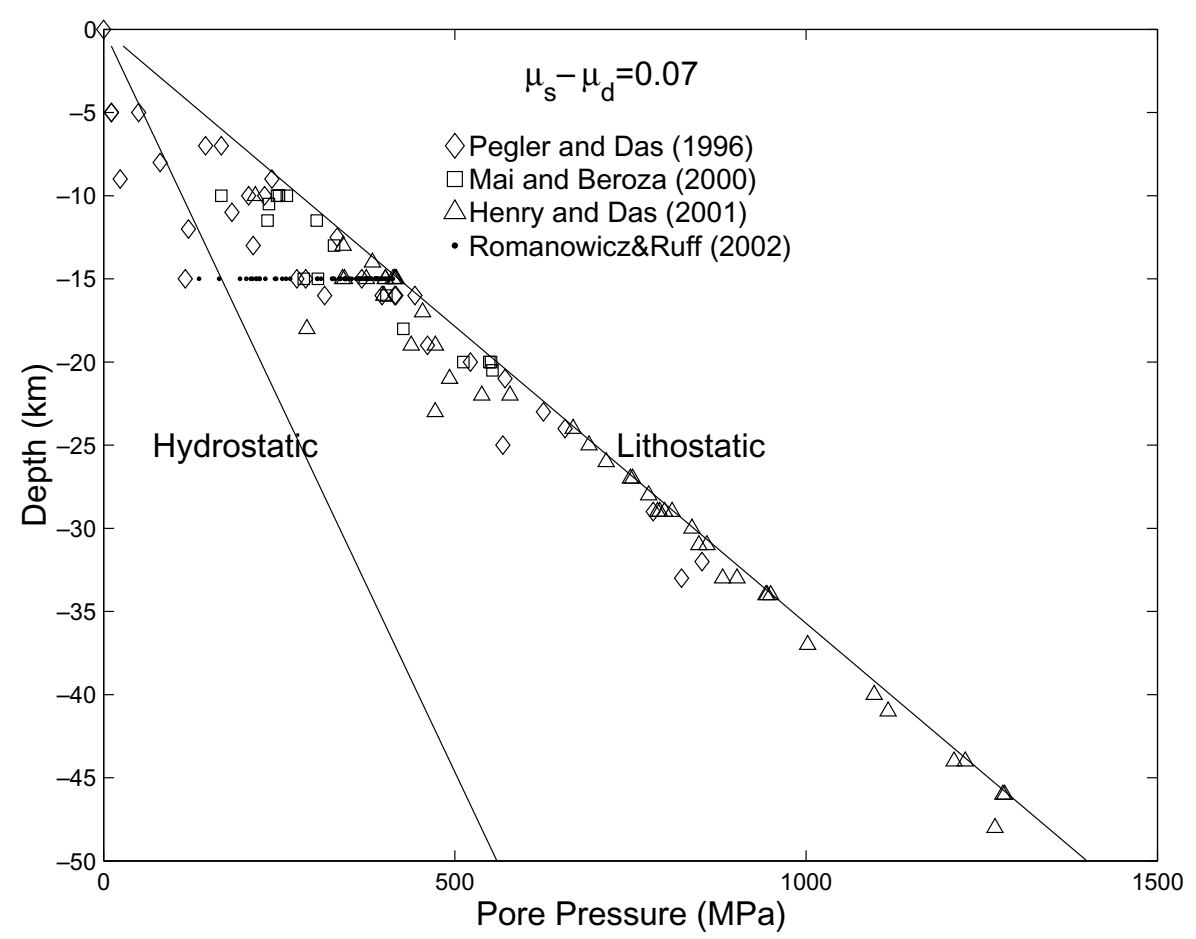

Fig. 3. Using $\lambda$ determined from Eq. (7) and assuming $\mu_{s}-\mu_{d}=0.07$. The pore pressure profiles show that pore pressures vary between hydrostatic and lithostatic up to a depth of about $15 \mathrm{~km}$, and a clear transition to near-lithostatic pore pressures below this depth. Higher values of $\mu_{s}-\mu_{d}$ show all large earthquakes fail as overpressured, weak faults. See also Miller (2002b).

In this explanation and the behavior of Eq. (5), the average slip increases with $L$ to a certain distance, but the influence of the rupture front subsides as the rupture grows large. As ruptures grow very large, slip evolves to a constant with in- creasing rupture length. Thus, ruptures exhibit L-model mechanics (e.g. $\bar{u} \propto L$ ) out to some distance, before converging to a constant slip model with increasing length. This is consistent with observations (Mai and Beroza, 2000), and is also 

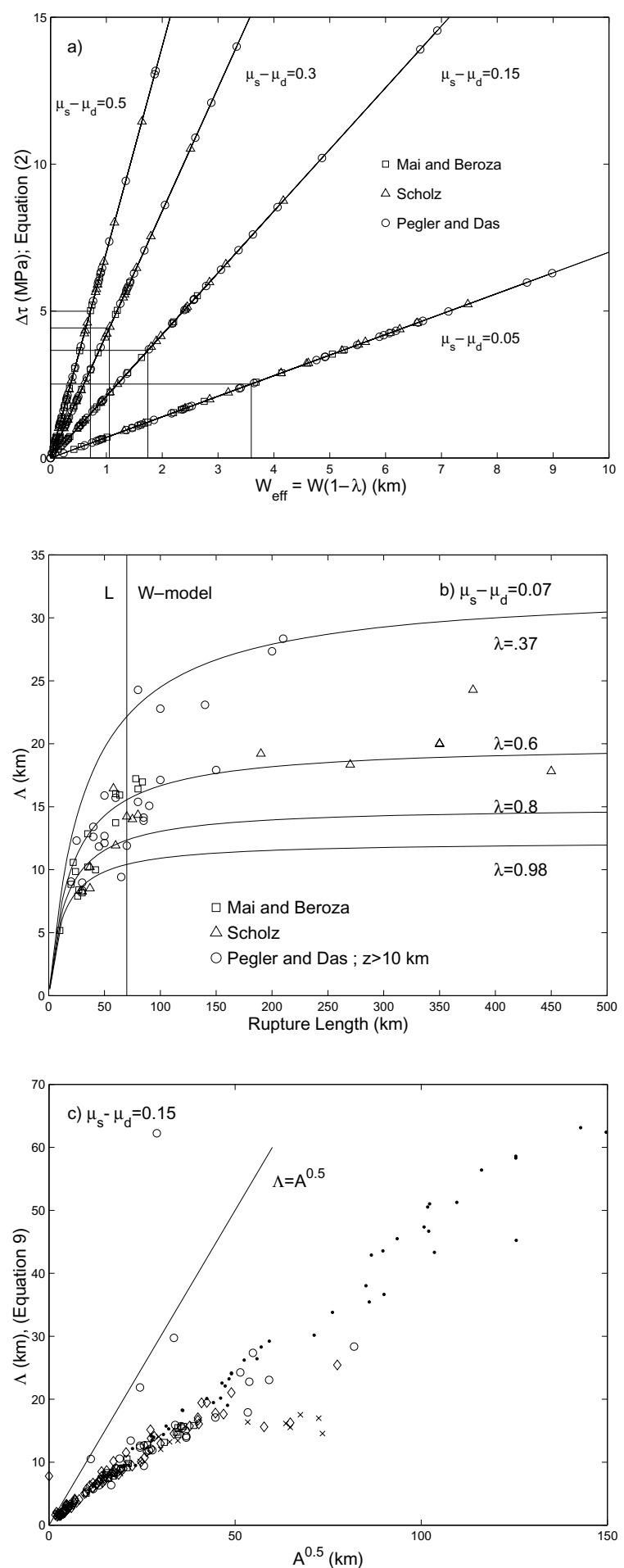

Fig. 4. (a) Using $\lambda$, the average effective width, $W_{\text {eff }}=W(1-\lambda)$, is only a few kilometers thick. The effective width can be viewed as the equivalent width of a dry, frictional fault. (b) $\Lambda$ as a function of rupture length showing the transition to constant slip is controlled by the fault overpressure. (c) Comparison of $\Lambda$ (Eq. (9)) with the traditional area scaling of large earthquakes.

consistent with a statistical analysis of strike-slip earthquake catalogs (Romanowicz and Ruff, 2002).

The primary support for the form of Eq. (5) results from the relationship between average slip and rupture length found from results of a numerical model of a propagating elastic dislocation (Miller, 2002a). Although these physical arguments and modeling results support the form of Eq. (5), additional study is needed to determine its validity. Assuming Eq. (5) to be valid, substitution of Eqs. (2) into (5) and using the seismic moment $M_{o}=G L W \bar{u}$ yields:

$$
M_{o}=\frac{\left(\mu_{s}-\mu_{d}\right) \rho_{r} g Z(1-\lambda) L^{2} W}{\left(1+\lambda \frac{L}{W}\right)}
$$

Fig. 1(a) shows the theoretical moment (Eq. (6)) superposed on the catalogs of strike-slip earthquakes (Pegler and Das, 1996; Mai and Beroza, 2000; Romanowicz and Ruff, 2002) for the case of a 15-km fault seismogenic width and different degrees of fault overpressure. Figure 1(a) shows that all 
of the data can be fit, but with a family of curves instead of either $M \propto L$ (W-model) or $M \propto L^{2}$ (L-model). This result is significant because it shows that the moment-length scaling argument (e.g. is it $L$ or is it $W$ ?) is not well-posed because the scatter in the data reflects fault overpressure instead of any fundamental scaling. No single line will fit the data because (as seen in Eq. (6)), the moment is a function of length, width and fault strength (e.g. overpressure). Figure 1(b) shows the moment-length data from model earthquake catalogs superposed on the natural earthquake catalogs to demonstrate model support for Eq. (6). Details of fault model are found elsewhere (Miller, 2002a).

Solving for $\lambda$ in Eq. (6),

$$
\lambda=1-\frac{1+\frac{W}{L}}{1+\frac{1}{M_{n d}}} ;
$$

where

$$
M_{n d}=\frac{M_{o}}{\left(\mu_{s}-\mu_{d}\right) \rho_{r} g Z W^{2} L} ;
$$

Equation (7) shows that pore pressures can be inferred by accurate measurements of the average rupture depth $(Z)$, the width $(W)$ and length $(L)$ of the rupture surface, and the scalar seismic moment $M_{o}$. Figure 2 shows the earthquake catalogs from different tectonic environments superposed on Eq. (7). The fit is of course guaranteed by using Eq. (7), but an independent check of the result is to use the values determined for $\lambda$ and the average depth of the event to calculate the pore pressure profiles (Fig. 3). The resulting profiles look very plausible and show that the pore pressure varies between hydrostatic and lithostatic above about $15 \mathrm{~km}$ depth, and a transition to near-lithostatic fluid pressure at depth.

Once $\lambda$ is known, other properties can be investigated. For the strike-slip earthquakes, using $\lambda$ determined by Eq. (7) in Eq. (2) (using $Z=W / 2$ ) shows that $\Delta \tau$ increases linearly with $W_{\text {eff }}$, where $W_{\text {eff }}=W(1-\lambda)$ can be viewed as the equivalent width of a dry, frictional fault. From a strength perspective, Fig. 4(a) shows that the average effective width of the seismogenic brittle crust is at most a few kilometers thick, depending of the choice of $\mu_{s}-\mu_{d}$. Furthermore, the average $\Delta \tau$ of the combined catalogs ranges between $2-$ $5 \mathrm{MPa}$, consistent with the historically referenced values for the average stress drop of large earthquakes (Scholz, 1990).

An implication of Eq. (5) is that the characteristic length $\Lambda$ in Eq. (2) has the form

$$
\Lambda=\frac{L}{\left(1+\lambda \frac{L}{W}\right)}
$$

and using $\lambda$ and knowing the rupture dimensions, $\Lambda$ can be estimated (Fig. 4(b)). When divided by the rupture velocity, $\Lambda$ provides a first-order approximation to the rise-times for these events. This is a result that can be tested by accurately measuring dislocation risetimes. The transition to $\mathrm{W}$-model mechanics at around $70 \mathrm{~km}$ (straight line) is consistent with observations (Romanowicz, 1992; Scholz, 1994; Mai and Beroza, 2000) that show a transition to W-model mechanics at very large rupture lengths. Implicit in the traditional area scaling $\left(M_{o} \propto A^{1.5}\right)$ scaling is that the characteristic length
$\Lambda=\sqrt{A}$. Figure 4(c) compares $\Lambda$ calculated with Eq. (4) with $\sqrt{A}$, and shows that for small earthquakes $\sqrt{A} \approx 2 \Lambda$, and diverges for larger events. Therefore, $\bar{u}$ determined from area scaling for large earthquakes can overestimate the average fault slip if Eq. (8) is a more appropriate form for $\Lambda$. Note that $\Lambda$ is dependent on the value chosen for $\mu_{s}-\mu_{d}$.

\section{Discussion}

The purpose of this paper is to argue that the relationship between the seismic moment of an earthquake and its rupture length depends on the strength of the fault that failed. Using heuristic arguments and support from a numerical earthquake model (Miller, 2002a), a form of the relationship between stress drop and average slip was proposed that includes the fault over-pressure as a parameter controlling the transition from L-model to W-model mechanics. The implication of the result is that faults appear to fail under a range of pore pressure, from near-hydrostatic pore pressures to near-lithostatic. A clear transition to near-lithostatic pore pressures is observed below a depth of about $15 \mathrm{~km}$, which supports the general intuition that rocks at depth can not fail through a stick-slip event when high pore pressures are absent. If faulting at large depths occurs under near-lithostatic pore pressure, then mechanisms must exist to generate fluid overpressures that dominate diffusive processes. Candidates include viscous compaction (Sleep and Blanpied, 1992), a fluid source at depth (Rice, 1992), or dehydration of hydrous minerals (Kirby, 1995; Ague et al., 1998; Peacock, 2001). If high fluid pressure is trapped behind a seal, then co-seismic rupturing of the seal will induce fluid flow if it then connects with sub-lithostatically pressured fluids. Thermodynamically, a reduction in fluid pressure has the same kinetic effect on dehydration as an increase in temperature, so can substantially accelerate the dehydration process and generate more fluid to drive the flow. This scenario is also supported by laboratory and modelling work (Miller et al., 2002c), and has significant implications for recycling of crustal fluids.

The mechanism for earthquake induced fluid flow is a coseismic change in permeability due to rupturing an impermeable seal. The breaking of the seal is simultaneous with dilatant slip, and allows near-lithostatic fluid pressures to communicate with sub-lithostatically pressured fluid in the overlying lithosphere. Co-seismic rupturing of an impermeable seal was the proposed explanation for tomographic observations following the July 30, 1995 Antofagasta earthquake in northern Chile $\left(M_{w}=8.0\right)$ (Husen and Kissling, 2001). This earthquake was a dip-slip subduction zone event with a rupture area about $240 \mathrm{~km}$ in length, $85 \mathrm{~km}$ in width, and an average depth of about $29 \mathrm{~km}$ (Henry and Das, 2001). Tomographic studies following this event showed spatio-temporal variations in $V_{p} / V_{s}$ (attributed to fluid flow), providing compelling evidence that earthquakes induce major changes in hydraulic conductivity and promote substantial fluid flow.

This scenario has been hypothesized to explain many earthquake related phenomena (Sibson, 1992; Muir-Wood and King, 1993; Miller et al., 1996; Roeloffs, 1998), and the evidence presented here for near-lithostatic fluid pressures at the time of large earthquakes lends further support for this mechanism. 


\section{Conclusion}

A variety of evidence from a range of fields shows that high pore pressures play a very important role in plate tectonic processes, and crustal hydraulics may be dominated by episodic flow. These ideas are not new, but here I attempt to quantify the degree of pore pressure associated with seismic faulting and show that the long-held notion of overpressured fluids deep in the crust are substantiated. Below about 15$20 \mathrm{~km}$, faults fail as overpressured, weak faults, resulting in an effective frictional depth of only a few kilometers (e.g. the equivalent depth of a dry, frictional fault). Evidence following large subduction zone earthquakes show that large scale fluid flow is triggered by the earthquake, consistent with models for seismic pumping and fault-valve behavior (Sibson, 1992). Additional modelling studies are needed to quantify crustal hydraulics, because the manner in which pore pressures diffuse following large seismic events have important implications on fluid-driven aftershocks, the kinetics of dehydration, and the initiation of melting through the rapid introduction of fluid.

Acknowledgments. I thank G. Beroza and an anonymous reviewer for insightful comments that significantly improved this manuscript. I also thank the organizers for the conference on 'Slip in and Below the Seismogenic Layer' held in Sendai, Japan. This is contribution No. 1252 of the Geophysics Institute.

\section{References}

Ague, J., J. Park, and D. Rye, Regional metamorphic dehydration and seismic hazard, Geophys. Res. Lett., 22, 4421-4224, 1998.

Blanpied, M., D. Lockner, and J. Byerlee, An earthquake mechanism based on rapid sealing of faults, Nature, 358, 574-576, 1992.

Caine, J., J. Evans, and C. Forster, Fault zone architecture and permeability structure, Geology, 24, 1025-1028, 1996.

Cochard, A. and R. Madariaga, Dynamic faulting under rate-dependent friction, Pure Appl. Geophys., 142, 419-445, 1994.

Cox, S., Faulting processes at high fluid pressures: An example of fault valve behavior from the Wattle Gully Fault, Victoria, Australia, J. Geophys. Res., 100, 12,841-12,859, 1995.

Evans, J. and F. Chester, Fluid-rock interaction in faults of the San Andreas system: Inferences from San Gabriel fault rock geochemistry and microstructures, J. Geophys. Res., 100, 13,007-13,020, 1995.

Floyd, J., J. Mutter, A. M. Goodliffe, and B. Taylor, Evidence for fault weakness and fluid flow within an active low-angle normal fault, Nature, 411, 779-783, 2001.

Fujii, Y. and M. Matsu'ura, Regional differences in scaling laws for large earthquakes and its tectonic implication, Pure Appl. Geophys., 157, 2283-2302, 2000.

Henry, C. and S. Das, Aftershock zones of large shallow earthquakes: Fault dimensions, aftershock area expansion, and scaling relations, Geophys. $J$. Int., 147, 272-293, 2001.

Husen, S. and E. Kissling, Postseismic fluid flow after the large subduction earthquake of Antofagasta, Chile, Geology, 29, 847-850, 2001.

Kirby, S., Intraslab earthquakes and phase changes in subducting lithosphere, Rev. Geophys., supp., 287-297, 1995.

Mai, P. and G. Beroza, Source scaling properties from finite fault rupture models, Bull. Seis. Soc. Am., 90, 604-615, 2000.

Manning, C. and S. Ingebritsen, Permeability of the continental crust: Implications of geothermal data and metamorphic systems, Rev. Geophys., 37, 127-150, 1999.

Matsu'ura, M. and T. Sato, Loading mechanism and scaling relations or large interplate earthquakes, Tectonophys., 277, 189-198, 1997.

Miller, S., The properties of large ruptures and the dynamical influence of fluids on earthquakes and faulting, J. Geophys. Res., 107, 10.1029/2000JB000032, 2002a.

Miller, S., Earthquake scaling and the strength of seismogenic faults, Geophys. Res. Lett., 29, 10.1029/2001GL014181, 2002 b.

Miller, S., A. Nur, and D. Olgaard, Earthquakes as a coupled shear stresshigh pore pressure dynamical system, Geophys. Res. Lett., 23, 197-200, 1996.

Miller, S., W. van der Zee, D. Olgaard, and J. Connolly, A fluidpressure controlled feedback model for dehydration reactions, Tectonophys., 2002c (in press).

Muir-Wood, R. and G. King, Hydrological signature of earthquake strain, $J$. Geophys. Res., 98, 22,035-22,068, 1993.

Peacock, S., Are the lower planes of double couple seismic zones caused by serpentine dehydration in subducting oceanic mantle?, Geology, 29, 299-302, 2001.

Pegler, G. and S. Das, Anaylysis of the relationship between seismic moment and fault length for large crustal strike-slip earthquakes between 1977-92, Geophys. Res. Lett., 23, 905-908, 1996.

Quattrocchi, F., In search of evidence of deep fluid discharges and pore pressure evolution in the crust to explain the seismicity style of the umbriamarche 1997-1998 seismic sequence Central Italy, Ann. Geophys., 42, 609-636, 1999 .

Rice, J., Fault stress states, pore pressure redistributions, and the weakness of the San Andreas fault, in Fault Mechanics and Transport Properties of Rock, edited by B. Evans and T.-F. Wong, pp. 476-503, Academic Press, 1992.

Roeloffs, E., Persistent water level changes in a well near Parkfield, California, due to local and distant earthquakes, J. Geophys. Res., 103, 869-889, 1998.

Romanowicz, B., Strike-slip earthquakes on quasi-vertical transcurrent faults-inferences for general scaling relations, Geophys. Res. Lett., 19, 481-484, 1992.

Romanowicz, B. and L. Ruff, On moment-length scaling of large earthquakes and the strength of faults, Geophys. Res. Lett., 29, 10.1029/2001GL014479, 2002.

Scholz, C., Scaling laws for large earthquakes: Consequences for physical models, Bull. Seismol. Soc. Am., 72, 1-14, 1982.

Scholz, C., The Mechanics of Earthquakes and Faulting, Cambridge Univ. Press, New York, 1990.

Scholz, C., A reappraisal of large earthquake scaling, Bull. Seis. Soc. Am., 84, 215-218, 1994.

Shaw, B. and C. Scholz, Slip-length scaling in large earthquakes: Observations and theory and implications for earthquake physics, Geophys. Res. Lett., 28, 2991-2994, 2001.

Sibson, R., Implications of fault-valve behavior for rupture nucleation and recurrence, Tectonophys., 211, 283-293, 1992.

Sleep, N. and M. Blanpied, Creep, compaction and the weak rheology of major faults, Nature, 359, 687-692, 1992.

Townend, J. and M. Zoback, How faulting keeps the crust strong, Geology, 28, 399-402, 2000.

Yin, Z.-M. and G. Rogers, Toward a physical understanding of earthquake scaling relations, Pure Appl. Geophys., 146, 661-675, 1996.

S. A. Miller (e-mail: steve.miller@erdw.ethz.ch) 